\title{
Modeling of C IV pumped fluorescence of Fe II in symbiotic stars
}

\author{
M. Eriksson ${ }^{1,3}$, H. Nilsson ${ }^{2}$, H. Veenhuizen ${ }^{1}$, and K. S. Long ${ }^{3}$ \\ 1 University of Kalmar, 39182 Kalmar, Sweden \\ e-mail: mattias@astro.lu.se \\ 2 Atomic Astrophysics, Lund Observatory, Box 43, 22100 Lund, Sweden \\ 3 Space Telescope Science Institute, 3700 San Martin drive, Baltimore, MD 21218, USA
}

Received 4 August 2007 / Accepted 12 September 2007

\begin{abstract}
Aims. We describe how the CIV $\lambda 1548.18$ line pumps the 1548.20 and $1548.41 \AA$ channels of Fe II in symbiotic stars through the process known as photo-ionization by accidental resonance (PAR). We describe where and why Fe II fluorescence arises in symbiotic stars and whether the Fe II $\lambda 1548.41$ channel can only be activated when there is a white-dwarf wind present in the system. Further, we aim to show how an analysis of the PAR-pumped lines helps to understand the phyisical conditions that they manifest.

Methods. We calculate intensities of the C IV-pumped Fe II fluorescence lines in symbiotic stars, corresponding to the $\mathrm{y}^{4} \mathrm{H}_{11 / 2}$ and $\mathrm{w}^{2} \mathrm{D}_{3 / 2}$ levels, based on a simple geometrical model of the emitting regions. We apply the model to seven symbiotic stars, known to have Fe II fluorescence lines pumped by C IV in their spectra. We compare the predicted intensities to the observed intensities of the selected symbiotic stars.

Results. We find that we can reproduce the observed fluorescence intensities of the seven symbiotic stars in our sample, using parameters that are consistent with their known properties. We show that PAR-produced lines can be used as a diagnostic tool to derive important physical parameters of a stellar system. We suggest that the detection of Fe II lines corresponding to the $w^{2} \mathrm{D}_{3 / 2}$ level in certain symbiotic stars provide evidence of hot shells expanding with velocities of at least hundreds $\mathrm{km} \mathrm{s}^{-1}$ in those systems.
\end{abstract}

Key words. binaries: symbiotic - radiation mechanisms: non-thermal - stars: atmospheres

\section{Introduction}

Symbiotic stars belong to a subclass of binary systems consisting of a hot compact object, usually a white dwarf, located within the stellar wind of its red giant companion. The UV spectra of symbiotic stars are characterized by many strong emission lines from gas in the wind from the red giant and its outer atmosphere ionized by the white dwarf radiation. The spectra display lines from a wide range of ionization states, from, for example, O I and C II to C IV, N V and O VI. They resemble, in many respects, those of planetary nebulae $(\mathrm{PNe})$ in terms of plasma conditions and abundances. However, the emission nebulae around symbiotic stars are less massive, and should have shorter lifetimes than those in PNe, since only a portion of the envelope of the red giant wind has been lost (Corradi et al. 1999).

The nebular regions of symbiotic stars are complex, and much of what we know about them is a result of observations with the IUE and subsequently HST and FUSE satellites. The emission-line spectra have contributions from the surrounding nebulae, the red giant and parts of the red giant atmosphere heated by the white dwarf radiation. The electron densities in symbiotic nebulae range from $10^{6}$ to $10^{11} \mathrm{~cm}^{-3}$ (Meier et al. 1994), and are considerably larger than those measured in PNe (Stanghellini \& Kaler 1989). Gradients in electron temperatures must be large in symbiotic stars to explain the wide range of ionization states. In some symbiotic systems, particularly in those which have recently undergone outbursts (Schmid 2000), there are winds originating from the vicinity of the white dwarf. In these cases, a collision between the fast white dwarf wind and slower red giant wind is inevitable. The existence of these collisions is directly observed at X-ray wavelengths
(Murset et al. 1997), but can also be inferred from profiles of some of the UV emission lines (Eriksson et al. 2004).

Emission lines are formed by many processes in symbiotic systems, and understanding which lines are formed by what process and under what physical conditions is key to understanding the structure of the nebular regions of symbiotic stars. Eriksson et al. (2006) analyzed over 600 UV emission lines in spectra of symbiotic stars and gave origins of formation as well as identifying the atomic process accounting for the population of the corresponding upper level for a majority of the lines. They concluded that a large fraction of relatively narrow Fe II lines present in UV spectra of symbiotic stars are formed by photo excitation by accidental resonance (PAR), a process originally described by Bowen (1935), and sometimes called Bowen-fluorescence. Specifically, Bowen (1935) showed that six O III multiplets at near UV and optical wavelengths in spectra of PNe could be explained in terms of an coincidence between the wavelengths of He II Ly $\alpha$ and O III $\lambda 303.799$ which pumped the upper levels of the UV/optical multiplets. Subsequently, Johansson (1983) showed that the presence of the conspicuous Fe II lines due to decays of the $\mathrm{y}^{4} \mathrm{H}_{11 / 2}$ level in the symbiotic stars RR Tel and V1016 Cyg could be explained by pumping of the $a^{4} \mathrm{~F}_{9 / 2}-\mathrm{y}^{4} \mathrm{H}_{11 / 2}$ channel by $\mathrm{C}$ IV $\lambda 1548.187$. To date, Fe II PAR pumped fluorescence lines have been found in 12 symbiotic stars (Eriksson et al. 2006). In some of these, more than $20 \mathrm{Fe}$ II channels are activated by various strong UV emission lines (Hartman \& Johansson 2000; Eriksson et al. 2004).

Most of the effort to understand the PAR-process in symbiotic stars has, to date, revolved around identifying the PAR-produced lines and understanding how effective the process 
is in producing such lines. Here we attempt to use the Fe II lines $\lambda 2479$ and $\lambda 2493$ in $I U E$ spectra of symbiotic stars to study the structure and nature of the nebulosity around individual systems. Measured fluorescence intensities from IUE spectra are compared to calculated intensities using a simple geometrical model in an attempt to determine the conditions in the fluorescence region of seven symbiotic stars. We view this as an exploratory step toward utilizing PAR produced lines as a diagnostic tool for studying symbiotic stars. A more detailed understanding of how the PAR produced lines correlate with the physics of its source could also be useful in analysis of other kinds of objects. As an example, PAR produced emission lines of Fe II are observed in AGN:s (Baldwin et al. 2004).

This paper is arranged as follows: in Sect. 2 we describe the model and discuss how fluorescence intensities are obtained, in Sect. 3 we describe the $I U E$ spectra and the information we have extracted to compare with the models. In Sect. 4 we describe our attempts to fit the observed intensities within the context of other known parameters for each system, and in Sect. 5, we summarize our results.

\section{Calculation of Fe II fluorescence line intensities}

The two ions, $\mathrm{Fe}^{+}$and $\mathrm{C}^{3+}$, that are involved in the PAR process described here have to be physically separated since the temperature and radiation environments required for their existance are very different. In symbiotic stars, the C IV region is located either in the atmosphere of the red giant heated by the white dwarf or in the central parts of a white-dwarf wind. In either case, the C IV region is small compared to the rest of the nebula. Since the purpose of the pumping region is to emit the C IV $\lambda 1548.2$ line it can be approximated as a point source. The Fe II region is thought to be located in part a of the circumstellar nebulosity while the specific shape of the region is not well defined, we can often estimate its size and location relative to the pumping region (see discussion of individual systems in Sect. 4). As a first attempt, we assume that the fluorescence region can be approximated as a sphere. Thus, the model geometry of a symbiotic system is described by two spheres. The spherical regions invoked in the modeling are:

- the pumping region, a point source of high ionization emitting C IV $\lambda 1548.187$ photons;

- the fluorescence sphere, containing $\mathrm{Fe}^{+}$ions that are pumped by the carbon $\lambda 1548.187$ line.

Again for simplicity, we assume that the $\mathrm{Fe}^{+}$spheres are homogeneous and have the same temperature and density throughout. The fluorescence sphere can be centered on the small pumping sphere in order to simulate shells of fluorescing material around the pumping region. Or, the fluorescing region can be set at some distance from the origin of pumping emission if only parts of the nebulae are assumed to be fluorescing.

The basic setup for the code is:

- the pumping sphere, having negligible radius, is located in the center of a selected coordinate system;

- the center of the fluorescence sphere is located at $\left(x_{0}, y_{0}, z_{0}\right)$ in the selected coordinate system, with radius $R$, temperature $T$, electron density $N_{\mathrm{e}}$, and number density of $\mathrm{Fe}^{+}$ions $N_{\mathrm{Fe}}$.

\subsection{The geometry}

In order to show the basic equations for calculation of the fluorescence line strengths, we first consider one fluorescence sphere, for which we select the coordinate system in such a way that the center of it coincide with the $x$-axis. The location and size of this sphere can then be described by the separation $x_{0}$ and the radius $R$ of the fluorescence sphere. Let $\theta$ be the angle between the propagation of an emitted C IV $\lambda 1548.187$ photon and the $x$-axis. The points where the photon enters and escapes the fluorescence sphere are then obtained from the equations:

$\left(x-x_{0}\right)^{2}+y^{2}+z^{2}=R^{2}$

$$
\left(y^{2}+z^{2}\right)^{1 / 2}=x \cdot \tan \theta,
$$

which have the solution:

$$
\begin{aligned}
& x=\frac{1}{1+(\tan \theta)^{2}} \cdot\left(x_{0} \pm \sqrt{R^{2}-\left(x_{0}^{2}-R^{2}\right)(\tan \theta)^{2}}\right) \\
& y=\frac{\cos \phi \cdot \tan \theta}{1+(\tan \theta)^{2}} \cdot\left(x_{0} \pm \sqrt{R^{2}-\left(x_{0}^{2}-R^{2}\right)(\tan \theta)^{2}}\right) \\
& z=\frac{\sin \phi \cdot \tan \theta}{1+(\tan \theta)^{2}} \cdot\left(x_{0} \pm \sqrt{R^{2}-\left(x_{0}^{2}-R^{2}\right)(\tan \theta)^{2}}\right)
\end{aligned}
$$

where $\phi$ is the rotation angle around the $x$-axis relative the $x y$-plane. The limit angle, $\tan \theta<\sqrt{\frac{R^{2}}{x_{0}^{2}-R^{2}}}$, corresponds to the angular size of the fluorescence sphere as seen from the pumping sphere. The path length $L$ through the fluorescence sphere for a photon can then be written as:

$$
\begin{aligned}
L(\theta)= & \frac{2}{1+(\tan \theta)^{2}} \\
& \times \sqrt{R^{2}-\left(x_{0}^{2}-2 \cdot R^{2}\right) \cdot(\tan \theta)^{2}-\left(x_{0}^{2}-R^{2}\right) \cdot(\tan \theta)^{4}} .
\end{aligned}
$$

Since the fluorescence sphere is homogeneous and of constant temperature and density, the line opacity of the Fe II channels is constant throughout the photon trajectories inside the sphere. The optical depth will hence be proportional to the length of the trajectory inside the fluorescence sphere.

After determining the sizes and locations of the spheres, i.e. defining the geometry, radial velocities relative to the pumping sphere can be assigned to the fluorescence sphere in order to define the dynamics of the system. Since the physics is assumed to be the same in the whole fluorescing region, the differences in optical depth for the C IV photons only depend on the relative length of their trajectories inside the sphere, and the relative velocity of the spheres.

\subsection{The fluorescing plasma}

The number of CIV $\lambda 1548.187$ photons, emitted between $\theta$ and $\theta+\mathrm{d} \theta$, entering and leaving the fluorescence sphere, $\left(\mathrm{d} n_{\mathrm{in}}\right)$ and $\left(\mathrm{d} n_{\text {out }}\right)$ respectively, are given by:

$\mathrm{d} n_{\mathrm{in}}=\frac{\sin \theta}{2} \cdot N_{\mathrm{ph}} \cdot \mathrm{d} \theta$

$\mathrm{d} n_{\text {out }}=\mathrm{d} n_{\text {in }} \cdot \mathrm{e}^{-\kappa \cdot L(\theta)}$

were $N_{\text {ph }}$ is the total number of C IV $\lambda 1548.187$ photons emitted by the pumping region. The number of excitations in the Fe II channels is then the difference between the entering and escaping photons. However, the number of photons produced due 
to fluorescence decay scales with the branching fractions $(B F)_{\mathrm{fl}}$ of the lines. Substituting the intensity of the pumping line $I_{\mathrm{p}}$ for the number of photons one obtains,

$\mathrm{d}\left(\mathrm{d} I_{\mathrm{fl}}\right)=(B F)_{\mathrm{fl}} \cdot I_{\mathrm{p}} \cdot \frac{\lambda_{\mathrm{p}}}{\lambda_{\mathrm{fl}}} \cdot \frac{\sin \theta}{2} \cdot\left(1-\mathrm{e}^{-\kappa(\lambda) \cdot L(\theta)}\right) \cdot \mathrm{d} \theta \cdot \mathrm{d} \lambda$

where $\mathrm{d}\left(\mathrm{d} I_{\mathrm{fl}}\right)$ is the intensity of fluorescence lines gained by the part of the C IV profile at wavelength $\lambda$, radiated at angle $\theta$ with respect to the line connecting the centers of pumping and fluorescence spheres. $\lambda_{\mathrm{p}}$ and $\lambda_{\mathrm{fl}}$ are the wavelengths of the pumping and fluorescence lines. The uncertainty in the absolute intensities will mainly depend on the errors associated with the distance to the objects, which is scaled away in Eq. (1). We will therefore set the peak intensity of the C IV line to one and express the integrated intensity of the Fe II lines in units of the peak intensity of the C IV $\lambda 1548$ line (we will hereafter use $\AA^{*}$ as notation for integrated fluorescence intensities expressed in terms of C IV $\lambda 1548$ peak intensities). The way the opacity is treated here is an improvement over earlier treatments of pumping efficiencies. Previously, the opacity for a pumping line entering a fluorescence region has been calculated by an overlap integral between the assumed profile of the injected pumping line and the profile of the fluorescence channel, which in most cases is obtained by assuming the same profile as for other absorption lines of the same ion (Kastner \& Bhatia 1991; Eriksson et al. 2005). In this way $\kappa(\lambda)$ becomes $\kappa$ and the integration over the wavelength is no longer necessary. Since the profile of the pumping line changes as it travels inside the fluorescence sphere, the overlap, and thus $\kappa$, cannot be constant. The logical error one makes by using the overlap integral is that the pumping photons are rearranged to a Gaussian distribution after each absorption by a fluorescent ion. In our treatment, the pumping line is divided into many lines defined by small wavelength intervals. By calculating a unique overlap integral for each of these pseudolines the accuracy of the pumping efficiency is greatly improved. The narrow components of the pumping line are sent individually at each specific trajectory of the pumping emission through the fluorescence sphere. At the end, the total excitations through the fluorescence channels are summed.

The opacity functions of the two pumping channels are calculated from the width of the Fe II lines, the electron density, the temperature and the number density of $\mathrm{Fe}^{+}$. For each system analyzed here, ranges of temperature and electron densities are given based on what is found in the literature. The number density of iron is poorly known in the nebulae of most symbiotic systems. In a few cases, we have made estimates of the iron abundance used for the calculations. Otherwise, the number density of iron is set at $10^{-5}$ to $10^{-4}$ of the electron density. We use the common LTE equations to derive the number densities of $\mathrm{Fe}^{+}$ions from the iron abundance, temperature and electron density. The exception is our use of the partition functions for which a new method of treatment is presented, which induces negligible errors from the Saha equation compared with the uncertainties induced by non-LTE effects.

\subsection{The atomic data}

For calculation of the ionization balance we used 380 Fe I levels, 350 Fe II levels, 328 Fe III levels, 260 Fe IV levels and $177 \mathrm{Fe} \mathrm{V}$ levels. The energies and statistical weights of the levels where obtained from NIST (NIST Atomic Spectra Database, NIST Standard Reference Database \#78) and references therein. The Fe II branching fractions and oscillator strengths used here
Table 1. Spectra used.

\begin{tabular}{lcccc}
\hline \hline System & $\begin{array}{c}\text { SWP } \\
\text { No. }\end{array}$ & $\begin{array}{c}\text { LWP } \\
\text { No. }\end{array}$ & $\begin{array}{c}\text { LWR } \\
\text { No. }\end{array}$ & $\begin{array}{c}\text { Noise at } 2485 \AA \\
10^{-13} \mathrm{erg} \mathrm{cm}^{-2} \mathrm{~s}^{-1}\end{array}$ \\
\hline EG And & 46879 & & 04717 & 1.62 \\
Z And & 04286 & 08389 & & 3.58 \\
V1016 Cyg & 24657 & 04961 & & 2.28 \\
RW Hya & 13601 & & 10227 & 1.24 \\
SY Mus & 46879 & 24737 & & 4.05 \\
RX Pup & 31285 & & 10831 & 1.56 \\
RR Tel & 18371 & & 16187 & 5.20 \\
\hline
\end{tabular}

are taken from the Kurucz database (Atomic spectral line database from CD-ROM 23 of R. L. Kurucz) and references therein.

The uncertainty in quantities in the involved Fe II levels are a source of concern for accurately calculating the fluorescent intensities. The parent-terms for the $\mathrm{y}^{4} \mathrm{H}_{11 / 2}\left({ }^{3} \mathrm{G}\right)$ and $\mathrm{w}^{2} \mathrm{D}_{3 / 2}\left({ }^{1} \mathrm{D} 2\right)$ correspond to Fe III levels with excitation energies between 3 and $4.5 \mathrm{eV}$. The high excitation energy of the $\mathrm{y}^{4} \mathrm{H}_{11 / 2}$ and $\mathrm{w}^{2} \mathrm{D}_{3 / 2}$ levels as well as the low interaction between levels with ${ }^{3} \mathrm{G}$ or ${ }^{1} \mathrm{D} 2$ parent terms and levels with the lowest Fe II parent term $\left({ }^{5} \mathrm{D}\right)$ makes it difficult to measure the required parameters of oscillator strengths. So far, oscillator strengths of Fe II $4 p$ levels have been measured to the fourth parent term $\left({ }^{3} \mathrm{~F} 2\right)$ at almost $8 \mathrm{eV}$ (Sikström et al. 1999). Ongoing work is being done to obtain lifetimes for levels of the fifth parent term $\left({ }^{3} \mathrm{G}\right)$, of which the $\mathrm{y}^{4} \mathrm{H}_{11 / 2}$ at $8.24 \mathrm{eV}$ is one. However, the oscillator strengths of the pumped levels discussed in this work, which belong to the fifth Fe II parent term, are derived from calculations.

\section{Symbiotic star spectra for comparison}

In order to compare the fluorescence intensities calculated with observed intensities, we have selected the IUE spectra of several symbiotic systems. The system characteristics are listed in Table 1. The SWP (short wavelength prime) spectra are in general chosen with short exposure times in order to avoid having saturated C IV lines. Strong C IV resonance lines easily saturate if one seeks to study the weaker lines of these systems. The LWP/LWR (long wavelength prime/redundant) spectra recorded with large apertures have been selected, with the longest exposure times possible since the Fe II fluorescence lines are quite weak in some of the systems and high $\mathrm{S} / \mathrm{N}$ level are needed to observe them.

Three of the parameters used in the calculation of fluorescence intensities are preferably obtained by measurements in observed spectra. These parameters are the peak intensity and width of the C IV $\lambda 1548$ line and the widths of Fe II lines. The C IV $\lambda 1548$ line is not measured directly, instead the peak intensity and width of the C IV $\lambda 1550$ is measured. Its peak intensity is assumed to be half that of C IV $\lambda 1548$ before it reached the Fe II ions and both lines are assumed to have the same widths. The reasons for employing this indirect method are given in Sect. 4. Further, the integrated intensities of the Fe II $\lambda 2493$ and $\lambda 2478$ lines are needed for comparison with the calculated values.

The width and peak intensity of the C IV $\lambda 1550$ line, $w_{1550}$ and $I_{\text {peak }}(\lambda 1550)$, are retrieved by fitting a Gaussian to the line in IUE short wavelength prime (SWP) high resolution spectra. The peak intensity of the C IV $\lambda 1548$ line, $I_{\text {peak }}(\lambda 1548)$, is two times $I_{\text {peak }}(\lambda 1550)$. Based on the FWHM of platinum lines, measured by Imhoff (1984), we have used a instrumental Gaussian 
Table 2. Fe II fluorescence and the C IV pumping lines in symbiotic stars.

\begin{tabular}{|c|c|c|c|c|c|c|c|}
\hline \multirow[t]{2}{*}{ System } & \multirow{2}{*}{$\begin{array}{c}I_{\text {peak }}(\lambda 1548) \\
\text { iu } \AA^{-1}\end{array}$} & \multirow{2}{*}{$\begin{array}{c}w_{\mathrm{CIV}} \\
\mathrm{km} \mathrm{s}^{-1}\end{array}$} & \multicolumn{2}{|c|}{$I_{2493}^{\mathrm{int}}$} & \multicolumn{2}{|c|}{$I_{2493}^{\mathrm{int}}$} & \multirow{2}{*}{$\begin{array}{c}\mathrm{W}_{\mathrm{Fe} \mathrm{II}} \\
\mathrm{km} \mathrm{s}^{-1}\end{array}$} \\
\hline & & & iu & $\AA^{*}$ & $\overline{\mathrm{iu}}$ & $\AA^{*}$ & \\
\hline EG And & 140 & 10.1 & 0.252 & 0.0018 & & & 11.4 \\
\hline Z And & 90 & 12.4 & 0.270 & 0.0030 & 0.068 & 0.0008 & 12.3 \\
\hline V1016 Cyg & 80 & 23.2 & 0.370 & 0.0046 & & & 11.2 \\
\hline RW Hya & 260 & 18.4 & 0.265 & 0.0010 & 0.105 & 0.0004 & 18.3 \\
\hline SY Mus & 95 & 57.7 & 0.125 & 0.0013 & & & 33.7 \\
\hline RX Pup & 95 & 56.2 & 0.380 & 0.0040 & & & 20.4 \\
\hline RR Tel & 480 & 26.8 & 0.703 & 0.0015 & 0.266 & 0.0006 & 9.7 \\
\hline
\end{tabular}

$\mathrm{iu}=10^{-12} \mathrm{erg} \mathrm{cm}^{-2} \mathrm{~s}^{-1}$. In units of the C IV $\lambda 1548$ peak intensity.

Table 3. Parameters for modeling symbiotic stars.

\begin{tabular}{lccccccccc}
\hline \hline System & $\begin{array}{c}T \\
\left(10^{3} \mathrm{~K}\right)\end{array}$ & $\begin{array}{c}N_{\mathrm{e}} \\
\log \left(\mathrm{cm}^{-3}\right)\end{array}$ & $\begin{array}{c}N_{\mathrm{Fe}} \\
\log \left(\mathrm{cm}^{-3}\right)\end{array}$ & $\begin{array}{c}R \\
(\mathrm{AU})\end{array}$ & $\begin{array}{c}D \\
(\mathrm{AU})\end{array}$ & $\begin{array}{c}I_{\text {wind }} \\
\left(I_{\text {neb }}\right)\end{array}$ & $\begin{array}{c}v_{\text {exp }} \\
\left(\mathrm{km} \mathrm{s}^{-1}\right)\end{array}$ & $\begin{array}{c}v_{\text {rel }} \\
\left(\mathrm{km} \mathrm{s}^{-1}\right)\end{array}$ & No $^{a}$ \\
\hline EG And $^{b}$ & $6-14$ & $7.1-9.1$ & $4.5^{c}$ & $0.5 \cdot \mathrm{D}$ & $0.7-7$ & 0 & 0 & 0 & 3 \\
Z And & $10-22$ & $7.0-10$ & $(4-5)^{c}$ & 2.0 & 0 & 0.03 & 300 & 0 & 3 \\
V1016 Cyg & 2.85 & $5.0-6.0$ & $3-6$ & 6.5 & $20-80$ & 0 & 0 & 0 & 3 \\
RW Hya & $6-12$ & 8.5 & 3.0 & 0.8 & 0 & $0-0.58$ & 170 & 0 & 2 \\
SY Mus & $2-20$ & $10-11$ & 3.4 & 2.5 & 2.5 & 0 & 0 & 20 & 2 \\
RX Pup & $2-20$ & $5.0-11$ & $5^{c}$ & $0-25$ & 25 & 0 & 0 & 20 & 3 \\
RR Tel & $11-15$ & 6.2 & 2.2 & $0-1000$ & 0 & 0.02 & 500 & 0 & 2 \\
\hline
\end{tabular}

${ }^{a}$ Number of varied parameters. ${ }^{b}$ The later calculated fluorescence intensities of EG And is divided by two in order to simulate a fluorescence region that is half of a circumstellar shell. ${ }^{c}$ The number density of iron is given as the logarithmic value of the fraction of the electron density.

width $\left(\sigma_{0}\right)$ of 0.13 to deconvolve the widths to be used for the $\mathrm{C}$ IV line in the FC calculations $\left(w_{1548}=\sqrt{w_{1550}^{2}-\sigma_{0}^{2}}\right)$. The peak intensities of the Fe II $\lambda 2493\left(I_{2493}^{\text {peak }}\right)$ and $\lambda 2479\left(I_{2479}^{\text {peak }}\right)$ lines are obtained by Gaussian fitting to the lines in IUE LWP or LWR high resolution spectra. Gaussian widths, $w_{i}$, are obtained by profile fitting for all unblended Fe II lines of good S/N level in the wavelength range of LWP. Then, the width used for the Fe II pumped channels in the FC calculation are obtained through:

$w_{\mathrm{Fe}}=\frac{1548.2}{k} \Sigma_{i=0}^{k} \frac{w_{i}}{\lambda_{i}}$

The integrated intensities of the two Fe II fluorescence lines are given by

$I_{\mathrm{c}}^{\mathrm{int}}=1.7725 \cdot \frac{\lambda_{\mathrm{c}}}{1548.2} \cdot w_{\mathrm{Fe}} \cdot I_{i}^{\mathrm{peak}}$.

The model we apply to symbiotic stars is a semi-empirical code in the sense that the C IV and Fe II Gaussian widths entering the calculations are directly measured from the spectra. The peak intensity of the CIV $\lambda 1548$ line is indirectly measured in the IUE spectra. Because the C IV $\lambda 1548$ line itself can be heavily effected by the PAR process and the peak intensity of the line before reaching the Fe II region is needed, the peak intensity used here is 2 times the measured peak intensity of the C IV $\lambda 1550$ line. This is an adequate method since deviations in the intensity fraction $I(\lambda 1548) / I(\lambda 1550)$ in fluorescing symbiotic stars mainly depend on the Fe II pumping by C IV (Eriksson et al. 2001).

Widths of Fe II and C IV spectra, as well as peak intensities of the C IV $\lambda 1548$ lines used for the symbiotic stars in our analysis are given in Table 2. In the same table the measured integrated intensity of the Fe II fluorescence lines, for which the calculated values are to be compared, are given.

\section{Modeling of symbiotic stars}

Although the model we apply to the PAR-produced lines of symbiotic stars is very simple, it still has a significant number (8) of free parameters that can in principle be varied. However, a number of parameters, in addition to the observed line intensities, are constrained by other studies of each of the symbiotic stars in our sample. We have used this information to limit the portion of the parameter space that we explore, and in particular to limit the number of parameters varied for each individual object. The parameters that were varied and the parameter space that was explored are summarized in Table 3. Specific explanations of the varied parameter choices are provided in the discussion of the model fitting of each star.

\section{1. $R R T e l$}

RR Tel is a well studied symbiotic star of the D (dusty) subclass in which the red giant is a long period Mira variable (Feast et al. 1989) which loses mass to the surrounding nebula, and a white dwarf of very high $(T>140000 \mathrm{~K})$ temperature (Jordan et al. 1994). Kotnik-Karuza et al. (2006) have recently estimated its distance to be $2.5 \mathrm{kpc}$. Although it is one of the most studied symbiotic systems, many of the physical parameters defining RR Tel are still uncertain, including the orbital period of the system. A better understanding of this system is needed in order to understand its extraordinarily diverse and rich emission line spectrum (Penston et al. 1983). RR Tel is one of the few known symbiotic novae, it erupted in 1945.

The appropriate electron density strongly depends on which lines are being used for the diagnostics. In this analysis we use the electron density obtained by Hayes \& Nussbaumer (1986) from the $\mathrm{Si}$ III] $I(\lambda 1883) / I(\lambda 1892)$ ratio which is $10^{6.2} \mathrm{~cm}^{-3}$, due to the relative temperature closeness needed for $\mathrm{Fe}^{+}$and $\mathrm{Si}^{2+}$. They also estimate the nebular temperature to be $13000 \pm 2000 \mathrm{~K}$, which is the range we use here. The number 
density used for iron in this analysis is $10^{2.2} \mathrm{~cm}^{-3}$ and is also based on solar $[\mathrm{Fe} / \mathrm{H}]$.

$\mathrm{RR}$ Tel is currently in a slow nova eruption state, and strong emission lines of highly excited levels show weak pedestals of Gaussian widths of approximately $500 \mathrm{~km} \mathrm{~s}^{-1}$. This gives us reason to assume that there is a white dwarf wind in RR Tel. The observed pedestals, when it is possible to resolve them, account for approximately $2 \%$ of the integrated flux of the lines (Harper et al. 1999). Hence, a white dwarf wind with an associated expansion velocity of $500 \mathrm{~km} \mathrm{~s}^{-1}$ emitting the C IV $\lambda 1548$ line with peak intensity such that its integrated intensity is $2 \%$ of the nebular (the C IV $\lambda 1548$ in the IUE spectra) flux is incorporated in our calculations.

At first, it seemed very difficult to define any geometry for RR Tel since neither the extent of any part of the nebula nor the binary orbit are known. However, from the parameters discussed thus far for this system, it is clear that the size of the fluorescence region must be large enough to explain the observed fluorescence intensities and the most logical assumption is that the $\mathrm{Fe}^{+}$ions lie in a shell around the system far from the central binary. This is reasonable given the high temperature of the white dwarf in the system. We have thus varied the distance to and the thickness of the fluorescence shell in modeling RR Tel.

Using the ranges for the parameters described in this section, it is impossible to come close the observed Fe II $I(2493) / I(2479)$ intensity ratio of 2.64 for $\mathrm{RR}$ Tel at temperatures higher than $11800 \mathrm{~K}$. Then, a grid of runs was made where the inner and outer radius of the fluorescence shell was varied from 50 to $1000 \mathrm{AU}$. The best match between the calculated and observed Fe II fluorescence line intensities was obtained when the outer radius of the shell was twice the inner radius. The thickness of the shell was therefore set to be the same as the radius of the enclosed space by the fluorescence region. Thus, there is only one free parameter describing the geometry of the shell. Figure 1 shows the calculated intensities of the two Fe II fluorescence lines as a function of the radius of the fluorescing shell. The observed Fe II 22493 intensity, $21 \times 10^{-13} \mathrm{erg} \mathrm{cm}^{-2} \mathrm{~s}^{-1} \AA^{-1}$, is reproduced for $x_{0}=490 \mathrm{AU}$ and the Fe II $\lambda 2478$ intensity, $8.0 \times 10^{-13} \mathrm{erg} \mathrm{cm}^{-2} \mathrm{~s}^{-1} \AA^{-1}$, is reproduced for $x^{0}=430 \mathrm{AU}$. Within the range $450 \pm 50 \mathrm{AU}$ the calculated values of both Fe II fluorescence lines are within the errors of the measured lines. The same interval for $x^{0}$ yields a calculated intensity fraction Fe II $I(2493) / I(2479)$ of 2.2 which is close to the observed value of 2.64, suggesting that the parameters chosen for the RR Tel model are reasonable.

\subsection{RW Hya}

RW Hya is an S-type symbiotic star, in which the WD is eclipsed by the M2 III red giant during a portion of its 370 day period (Kenyon \& Mikolajewska 1995). Compared to most symbiotic stars, for RW Hya the physics of the system are relatively well known and hence, the ranges of physical properties in the literature have narrower ranges. The red giant has a mass of $1.6 M_{\odot}$, a radius of $0.27 \mathrm{AU}$ and a total luminosity of $600 L_{\odot}$. The WD has a mass of $0.5 M_{\odot}$ (Schild et al. 1996).

The RW Hya nebula is compact and estimates of the size yield a radius of approximately 0.5 AU (Schild et al. 1996). Dumm et al. (1999) determined that the mass loss of the red giant wind corresponds to $5 \times 10^{-7} M_{\odot}$ per year. They further measured the temperature of the red giant wind to be about $10000 \mathrm{~K}$. From line blanketing of Fe II they suggest the number density of ions is $\sim 3 \times 10^{8} \mathrm{~cm}^{-3}$, which using solar element concentrations

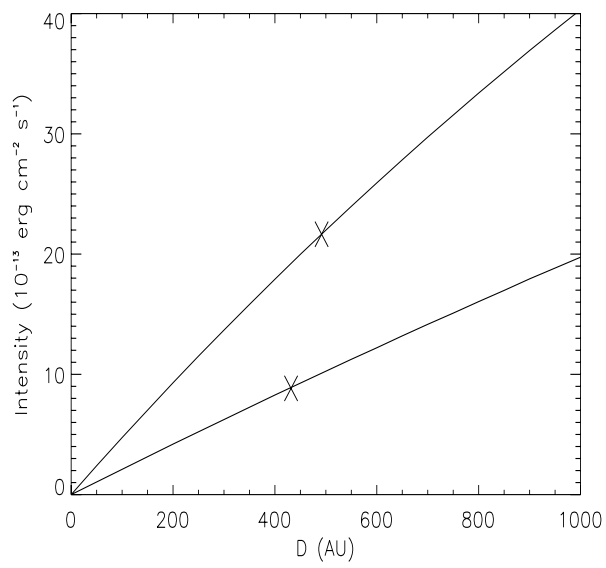

Fig. 1. RR Tel, the intensity of the Fe II $\lambda 2493$ line (solid line) and $\lambda 2479$ line (dashed) as a function of the extension of the fluorescence shell around the binary. The marks are the observed intensities placed on the respective calculated functions.

corresponds to a relative iron density $n_{\mathrm{Fe}}=10^{3} \mathrm{~cm}^{-3}$. The nebular electron density in RW Hya is about $10^{9} \mathrm{~cm}^{-3}$ (Kenyon \& Fernandez-Castro 1987).

The C IV resonance doublet in IUE spectra is strong and narrow and originates from part of the red-giant wind irradiated by the white dwarf. Dumm et al. (2000) measured an increase of the column density of hydrogen at phase 0.78 . Their explanation of this increase is a high density wake caused by shocks from accretion of the red giant wind onto the white dwarf at supersonic speed. In addition, from the UV emission line profile, the white dwarf in RW Hya has been reported to expel a wind of $170 \mathrm{~km} \mathrm{~s}^{-1}$ (Sion et al. 2002). Since it is not known if there is a white dwarf wind in RW Hya, we do not know if there is any contribution of such a wind to the C IV pumping line. No such contribution to the C IV $\lambda 1548$ emission line has yet been observed in RW Hya. From profile fitting of the C IV line in the IUE spectra SWP13601 it is concluded that the maximum peak intensity of a $170 \mathrm{~km} \mathrm{~s}^{-1}$ broad contribution is $1.5 \times 10^{-11} \mathrm{erg} \mathrm{cm}^{-2} \mathrm{~s}^{-1} \AA^{-1}$.

In calculating the RW Hya fluorescence intensities, we have fixed the number density of electrons and iron ions to those described above. The nebula of RW Hya consists of a red giant wind which encloses the binary in a compact ionized shell. From 0.2 to $0.8 \mathrm{AU}$ from the center of the white dwarf, $\mathrm{Fe}^{+}$ions are pumped resulting in the Fe II fluorescence lines. Temperatures between 6000 and $12000 \mathrm{~K}$, in $100 \mathrm{~K}$ steps, have been tested for the nebulae. Except for the temperature, the only parameter allowed to vary was the peak intensity of the $170 \mathrm{~km} \mathrm{~s}^{-1}$ wide plausible wind contribution of the C IV $\lambda 1548$ line. Fifteen different peak intensities from 0 to $1.5 \times 10^{-11} \mathrm{erg} \mathrm{cm}^{-2} \mathrm{~s}^{-1} \AA^{-1}$ were tested for each temperature giving a grid of 900 calculations.

In the absence of C IV emission from the wind of the white dwarf it is impossible to reproduce the observed Fe II fluorescence intensities within reasonable deviations (Fig. 2). This suggests that the white dwarf has a wind that is hidden to a distant observer. In order to simultaneously obtain the observed intensities of the Fe II $\lambda 2493$ and $\lambda 2478$ as well as their relative intensity within a factor of 2 , the peak intensity of the wind emission must be higher than $0.9 \times 10^{-11} \mathrm{erg} \mathrm{cm}^{-2} \mathrm{~s}^{-1} \AA^{-1}$. This is close to the observational upper limit of $1.5 \times 10^{-11} \mathrm{erg} \mathrm{cm}^{-2} \mathrm{~s}^{-1} \AA^{-1}$. The temperature interval for which the spectral parameters are reproduced within a factor of two is $7800-8600 \mathrm{~K}$. 


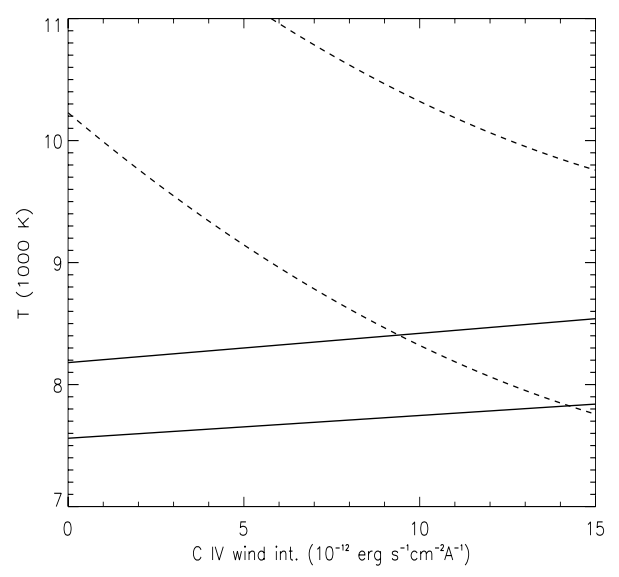

Fig. 2. Temperature versus peak intensity of the C IV wind emission in RW Hya. The Fe II $\lambda 2478$ intensity can only be satisfactorily reproduced for temperatures between the two solid lines. The Fe II intensity ratio $I(\lambda 2493) / I(\lambda 2478)$ can only be satisfactorily reproduced for temperatures above the dashed line. We define "satisfactorily reproduced" as matching observations within a factor of 2 . This graph confirms the presence of a white dwarf wind, already mentioned by Sion et al. (2002) and suggests a temperature for the fluorescent part of the nebula of RW Hya of $8200 \pm 400 \mathrm{~K}$.

\subsection{Z And}

The light curve of $Z$ And has probably been studied more extensively than any other symbiotic star. Three different periodicities have been observed, including the 759 day orbital period of the system (Formiggini \& Leibowitz 1994). Assuming solar masses for the two components, the separation between the two stars is approximately $2 \mathrm{AU}$. The source of most of the emission lines in $\mathrm{Z}$ And is a wind from the red giant ionized by the UV radiation from the white dwarf which forms a bow like nebula around the red giant (Fernandez-Castro et al. 1988). The fluorescence region is then best represented as a shell around the pumping emission. In our model the fluorescence region is represented by a number of spheres, together forming a shell around the system at a distance of 0.1 to $1.9 \mathrm{AU}$.

Electron densities for the various regions in $\mathrm{Z}$ And are difficult to obtain with high precision. The electron density in the ionized surrounding nebula has been measured to $\sim 10^{10} \mathrm{~cm}^{-3}$ with He I $I(6678) / I(5875)$ and $I(7065) / I(5875)$ line ratios (Proga et al. 1994). But forbidden lines at optical wavelengths, such as [O III], yields $\sim 10^{8} \mathrm{~cm}^{-3}$ (Ferland \& Shields 1978). Because of the large uncertainty of electron density, a wide range of electron densities from $10^{7}$ to $10^{10} \mathrm{~cm}^{-3}$ have been tested in calculating the Fe II fluorescence intensities. No satisfactory value is given in the literature of the number density of iron, therefore for each electron density we have tested a range of relative iron abundance $\left(n_{\mathrm{Fe}} / n_{\mathrm{e}}\right)$ from $10^{-4}$ to $10^{-5} \mathrm{~cm}^{-3}$.

$\mathrm{Z}$ And is a relatively compact object, at least compared to V1016 Cyg. Therefore a substantial temperature gradient is expected in the surrounding nebula. This is the most likely the explanation for the large variations in electron temperatures obtained for the system. Although the temperature varies between 10000 and $30000 \mathrm{~K}$, the mean value is $20000 \mathrm{~K}$ (Mikolajewska $\&$ Kenyon 1996). From our fluorescence code it can be shown that it is impossible to produce the $\lambda \lambda 2479,2493$ lines in plasma defined by the parameters for $\mathrm{Z}$ And at temperatures above $22000 \mathrm{~K}$. We have thus modeled temperatures between 10000 and $22000 \mathrm{~K}$.
Since the majority of the lines, among them the Fe II fluorescence lines, originates from an expansive region, the widths of the lines are larger than in most symbiotic stars. As the Fe II lines in Z And are unusually broad, the C IV lines are at a first glance exceptionally narrow. The best Gaussian fit to the C IV $\lambda 1550$ line in the lwp08389 spectrum gives a width of only $0.064 \AA$ (Instrumental profile of $0.12 \AA$ ). However, it is not possible to fit a Gaussian to the C IV line very well since there seems to be an extra broad emission feature at the base of the nebular line. The $\mathrm{S} / \mathrm{N}$ level of the IUE spectrum does not allow determination of the nature of this light. However, in a careful investigation of the ultraviolet spectrum of $Z$ And, Nussbaumer \& Vogel (1989) concluded that a white dwarf wind of $\sim 300 \mathrm{~km} \mathrm{~s}^{-1}$ can explain the broad emission features in the spectrum.

The maximum peak intensity, based on the noise level in the lwp08389 spectrum, of the C IV $\lambda 1548$ line emitted by the white dwarf wind is 0.056 times the intensity of the narrower nebular line $\left(I_{\mathrm{p}}\right)$. Intensities from zero to the maximum intensity are tested in our code. The terminal velocity of the white dwarf wind is set to $300 \mathrm{~km} \mathrm{~s}^{-1}$.

In the selected $\left(n_{\mathrm{e}}, n_{\mathrm{Fe}}, T\right)$ parameter space only a narrow range of C IV intensities is possible. For wind-intensities below $0.025 I_{\mathrm{p}}$, the Fe II $\lambda 2479$ would be unobservable and for intensities above $0.042 I_{\mathrm{p}}$ the $I(2493) / I(2478)$ fraction is below the observed value of 4.0 for the whole parameter space. Thus, the model strongly predicts that with data of at least 5 times the $\mathrm{S} / \mathrm{N}$ of $I U E$ it would be possible to observe the white dwarf wind emission directly, just as in for example AG Peg (Eriksson et al. 2004). In the investigation of the other free parameters, the white dwarf wind emission was set to $0.033 I_{\mathrm{p}}$.

In order to have enough Fe II fluorescence intensity to be observed with IUE the electron density required is $2 \times 10^{9} \mathrm{~cm}^{-3}$. The calculations also give limits on the temperature (Fig. 4) which must be less than $13000 \mathrm{~K}$ in order for the Fe II fluorescence to be observable. In Fig. 4 it can be seen that for electron densities above $2 \times 10^{9} \mathrm{~cm}^{-3}$ in the fluorescence sphere the temperature must exceed $11000 \mathrm{~K}$ in order to obtain the same $I(2493) / I(2478)$ ratio in the calculations as in the observed spectrum. Hence, taking into account the temperature gradient in the nebula, the $\mathrm{Fe}^{+}$ions that are pumped by the $\mathrm{C}$ IV emission must be at a distance from the system where the nebular temperature is between 11000 and $13000 \mathrm{~K}$.

\section{4. $R X$ Pup}

IUE spectrum of RX Pup has a strong Fe II $\lambda 2493$ fluorescence line $\left(0.0040 \AA^{*}\right.$, where the unit $\AA^{*}$ is explained in Table 2). The pumping has to be very effective in order to explain such Fe II fluorescence lines.

In IUE spectra of RX Pup observed during the 1980s the CIV intensity ratio $I(1548) / I(1550)$ had values less than unity. Michalitsianos et al. (1988) suggested that the anomalous intensity ratio could be explained by a temporary outflow from the white dwarf. However, calculations by Eriksson et al. (2001) shows that the deficient flux in the C IV $\lambda 1548$ line matches what has been lost due to the Fe II pumping. Because of the low C IV emission intensity in RX Pup (relative to other symbiotic stars), a large fraction of the C IV $\lambda 1548$ line must be pumping Fe II in order to raise the Fe II fluorescence lines above the noise in the IUE spectra. In our model of RX Pup we do not assume any white dwarf wind.

The geometrical properties of RX Pup still have large uncertainties. An early estimate of the binary separation was $17 \mathrm{AU}$ 
(Ivison \& Seaquist 1994). This was difficult to reconcile with estimates of the radius of a permanent dusty Mira shell at $\sim 77 \mathrm{AU}$ (Anandarao et al. 1988). The issue of the RX Pup geometry is discussed by Mikolajewska et al. (1999), where the binary separation is suggested to be more than $50 \mathrm{AU}$. In our model we have utilized a binary separation of 50 AU. The Mira shell extends half way to the white dwarf which makes the radius $25 \mathrm{AU}$. The fluorescence takes place in the part of the Mira shell closest to the white dwarf. As the position is locked in this way, a grid of radii for the fluorescence region is tested.

Electron densities in the plasma in the system seem to vary much with location. Values from $10^{7} \mathrm{~cm}^{-3}$ (Whitelock et al. 1984) derived by optical forbidden lines to $10^{10} \mathrm{~cm}^{-3}$ (Kafatos et al. 1982) derived from UV inter combination lines have been reported. The electron temperature in the fluorescence region is also difficult to estimate since the nebula consists of typically low temperature regions (the inner parts of the Mira shell) as well as regions of temperatures in excess of $15000 \mathrm{~K}$ (Mikolajewska et al. 1999). Because of the uncertainties regarding density and temperature a large grid of electron densities (from $10^{5}$ to $10^{11} \mathrm{~cm}^{-3}$ ) and temperatures (from 2000 to 20000 ) is tested in this work.

Outflows from the system of $\sim 10^{-6} M_{\odot}$ are associated with a $20 \mathrm{~km} \mathrm{~s}^{-1}$ expansion velocity of the MIRA shell (Allen 1988). Since the fluorescence region in our model of RX Pup is the part of the Mira shell closest to the white dwarf, a relative velocity of $-20 \mathrm{~km} \mathrm{~s}^{-1}$ is used for the line strengths.

The Fe II $\lambda 2493$ line has an integrated measured intensity of $7 \times 10^{-13} \mathrm{erg} \mathrm{s}^{-1} \mathrm{~cm}^{-2}$. Since the total energy output of this line in the fluorescence region depends on the size of the fluorescent region, which is poorly known, there is no purpose in matching the calculated intensity to the observed intensity. However, the possible $\left(T_{\mathrm{e}}, n_{\mathrm{e}}\right)$ space for the fluorescent region can be quite well determined by looking at the order of magnitude of the theoretical $\lambda 2493$ intensities. In order to have observable Fe II fluorescence lines, the allowed range of temperature has to be 3000 to $7000 \mathrm{~K}$ and the electron density has to be $10^{9}$ to $10^{11} \mathrm{~cm}^{-3}$ (Fig. 5). Within the possible $\left(T_{\mathrm{e}}, n_{\mathrm{e}}\right)$ space, due to the Fe II $\lambda 2493$ line, there exists no point for which the Fe II w ${ }^{2} \mathrm{D}_{3 / 2}$ level is populated enough to produce observable lines (Fig. 3). This is consistent with the lack of the Fe II $\lambda 2479$ line in the IUE spectrum of RX Pup.

\subsection{SY Mus}

The cool component of SY Mus is a M4 red giant star with $R=86 R_{\odot}$ (Schmutz et al. 1994). The period of the binary system is 624.5 days (Pereira et al. 1995) which implies a binary separation $\sim 4$ AU. Most of the observed nebulosity of SY Mus is believed to be due to the red giant wind which is ionized by the hot white dwarf companion. Different parts of such a wind would be subjected to very different radiation fields, depending on the location relative to the white dwarf. A complex ionization structure is therefore expected. Previous works concerning this red giant wind have given a good idea of the basic nature of the wind. Starting at one radius $(R)$ distance from the red giant, the wind accelerates from almost zero to almost terminal velocity $\left(v_{\infty}\right)$, which is $20 \mathrm{~km} \mathrm{~s}^{-1}$, at approximately $2.5 R$ (Dumm et al. 1999). Since there is no sign of Fe II P Cygni profiles and the Fe II lines are too narrow to be formed in an optically thin accelerating wind the Fe II fluorescing region must be outside the accelerating part of the red giant wind.

The He II emission lines seen in SY Mus spectra come from a small sphere around the white dwarf (Pereira et al. 1995). This

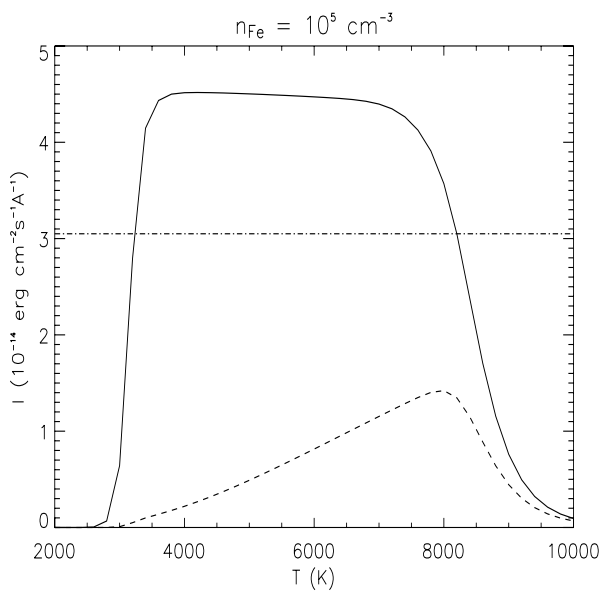

Fig. 3. The intensity of the Fe II fluorescence caused by C IV pumping for the parameters, whose ranges are specified in the text, giving the strongest Fe II fluorescence lines. The solid and dashed lines correspond to the intensity of the $\lambda 2493$ line and the $\lambda 2479$ line, respectively. The noise level in the used IUE spectra is given as the straight line in the figure.

small sphere is also most likely the origin of other ions of high ionization energy. In the SY Mus geometry adopted here, the pumping sphere is a bubble of highly ionized material in the red giant wind at the location of the white dwarf. The fluorescence region begins close to the pumping region, without any overlap, in the direction of the red giant and stretches all the way to where the accelerating part of the red giant wind ends (2.5 AU from the white dwarf). In the directions perpendicular to the white dwarf - red giant axis the fluorescence region stretches 2.5 AU in all directions.

Since the nebula is dominated by the red giant wind the density at distances less than the binary separation should depend only on the distance to the red giant itself. Given a mass loss rate $\dot{M}=10^{-9} M_{\odot}$ (Pereira et al. 1999) and assuming a solar mass fraction of iron $\mu=0.001287$ (Grevesse \& Sauval 1999) it is possible to calculate the number density of iron in the fluorescing region of SY Mus, of

$n_{\mathrm{Fe}}=\frac{\dot{M} \cdot \mu}{4 \cdot \pi \cdot v \cdot m_{\mathrm{Fe}} \cdot D^{2}}$.

The derived number density of iron in the fluorescing region using Eq. (4) is $2.5 \times 10^{3} \mathrm{~cm}^{-3}$. As for many of the symbiotic stars, the SY Mus UV spectrum shows no lines corresponding to the magnetic quadrapole transitions belonging to the ${ }^{1} \mathrm{~S}^{e}-{ }^{3} \mathrm{P}^{\circ}$ multiplets in C III or Si III while the allowed intercombination of the same transitions are among the strongest lines in the entire spectra. Usually, this directly indicates electron densities above $10^{6} \mathrm{~cm}^{-3}$. Based on diagnostics developed by Nussbaumer (1987), where I(Si III]):I(C III]) regarding the E1 ${ }^{1} \mathrm{~S}-{ }^{3} \mathrm{P}$ transitions are used to determine electron densities, Pereira et al. (1995) estimated the density in the SY Mus plasma to be $10^{10} \mathrm{~cm}^{-3}$ or slightly higher. Temperature estimates in nebulae of complex structure such as the SY Mus nebula strongly depend on the lines used. No temperature estimates so far in the literature can, with confidence, be assigned to the fluorescing region. Therefore we constructed models with electron temperatures in the range of 2000 to $20000 \mathrm{~K}$ and electron densities between $10^{10}$ and $10^{11} \mathrm{~cm}^{-3}$ to span a large parameter space.

For all choices of temperature, the intensity of the Fe II $\lambda 2479$ line is far below the detection limit in the IUE spectra. This is consistent with the fact that no traces of Fe II w ${ }^{2} \mathrm{D}_{3 / 2}$ 

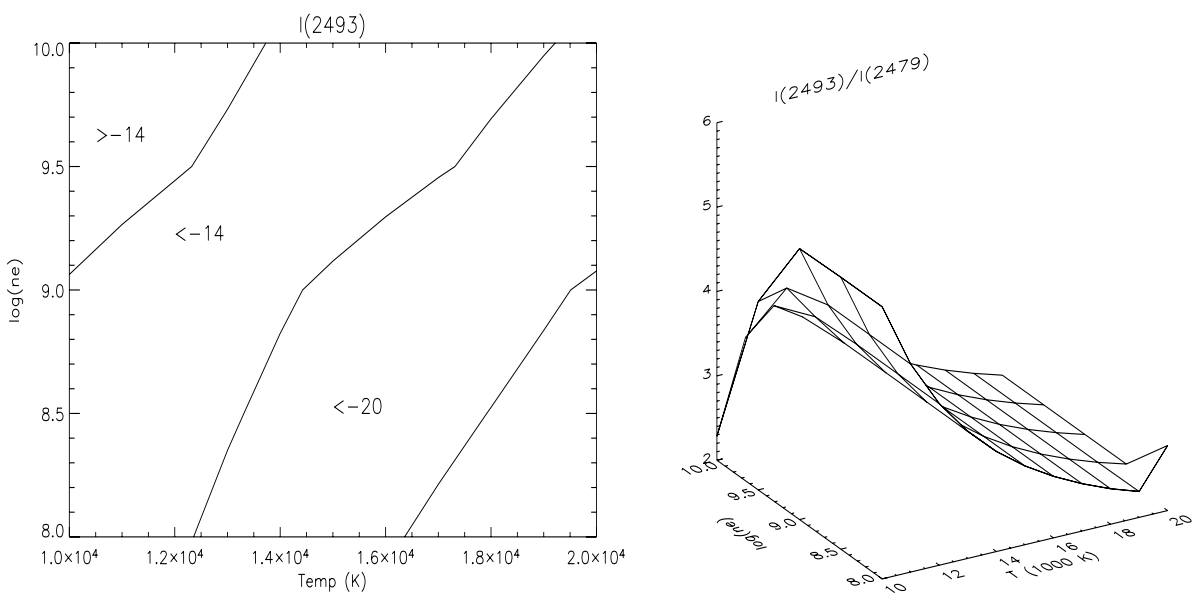

Fig. 4. Z And, left panel shows the intensity of the Fe II $\lambda 2493$ line and right panel the $I(2493) / I(2478)$ ratio as a function of electron density and temperature. In both plots the number density of iron is set to $10^{-4.5}$ times that of the electron density. The white dwarf wind contributes a $3 \times 10^{-12} \mathrm{erg} \mathrm{cm}^{-2} \mathrm{~s}^{-1} \AA^{-1} \mathrm{C}$ IV $\lambda 1548$ line of $300 \mathrm{~km} \mathrm{~s}^{-1}$ width plunging into the fluorescence region.
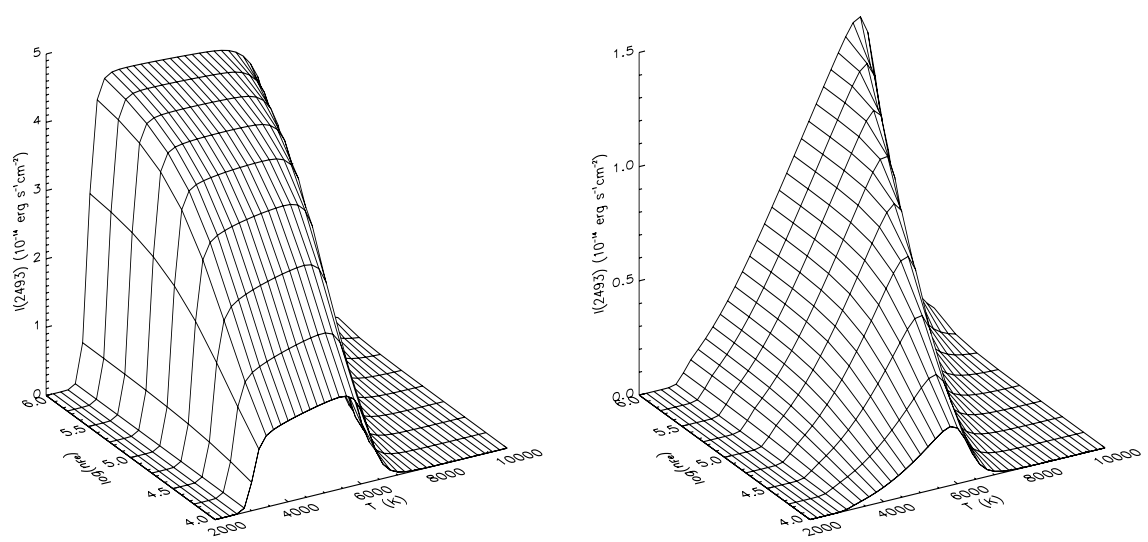

Fig. 5. Integrated intensity of the Fe II fluorescence lines $\lambda 2493$ and $\lambda 2479$ lines as given by the code for RX Pup. According to the calculations, and the observations of Fe II $\lambda 2493$ in IUE spectra, the temperature of the region where the $\mathrm{Fe}^{+}$gets pumped must be between 3000 to $7000 \mathrm{~K}$ and number density of iron must be above $10^{4.5} \mathrm{~cm}^{-3}$. In agreement with observations the calculated intensity of the $\lambda 2479$ line is lower than what is observable with IUE.

radiation have been observed in SY Mus spectra. Fluorescence lines from the $\mathrm{y}^{4} \mathrm{H}_{11 / 2}$ level are observed in SY Mus spectra and the integrated intensity of the $\lambda 2493$ line is $1.25 \times$ $10^{-13} \mathrm{erg} \mathrm{cm}^{-2} \mathrm{~s}^{-1}$. According to the best parameter fit (Fig. 6) this line intensity can be reached for temperatures between 4600 and $6800 \mathrm{~K}$. However, based on the uncertainties of the calculated intensities the temperature limits we give are between 4000 and $7500 \mathrm{~K}$. Still, it is interesting that modeling of fluorescence lines can give estimates on temperature.

\subsection{EG And}

EG And is composed of a somewhat complex wind structure. P-Cygni profiles from UV spectroscopy indicate fast outflows and multiple line components indicate various wind domains as emission sources (Eriksson et al. 2006). At the same time as the white dwarf seems to accrete matter from the red giant wind at $8.1 \times 10^{-8} M_{\odot}$ per year, the overall system loses matter with a speed of $3.3 \times 10^{-7} M_{\odot}$ per year (Skopal 2005). Even though EG And seems to be a binary containing an accreting white dwarf, the models developed so far do not indicate any disc around the white dwarf (Kolb et al. 2004). We still do not understand the nature of the driving forces of the wind structures in this system.

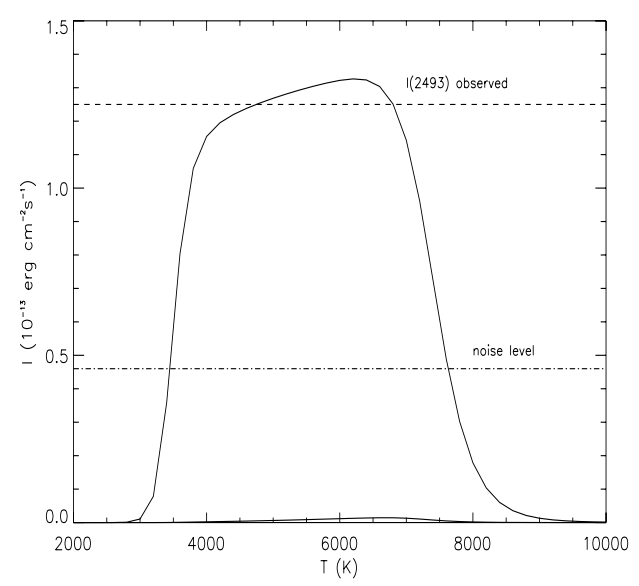

Fig. 6. The intensity of the Fe II fluorescence lines in SY Mus as a function of the temperature within the fluorescence region.

Both stars in the EG And system share a common envelope which is co-rotating with the central binary (Skopal et al. 1993). This common envelope is built up by the red giant wind. The radiation from the white dwarf is partly blocked by this envelope (the extent of UV emission passing the envelope depends on the phase of EG And) mainly by Raleigh scattering of both 
atomic and molecular hydrogen (Vogel 1991). The Fe II fluorescence lines in the EG And spectrum are formed at the depth in the common envelope where the temperature is sufficient to create $\mathrm{Fe}^{+}$ions. The pumping region in EG And is located at the top of the red giant atmosphere from which the C IV $\lambda 1548$ line can reach half of the surrounding Fe II fluorescence shell. The general properties of the model of EG And emphasize a small pumping sphere at origo, radiating only in the positive $x$-direction, pumping $\mathrm{Fe}^{+}$ions in half of the common envelope.

The central binary in EG And is not resolved. The orbital period $(P)$ of the system has been measured with UBV variations to 482.6 days (Tomov \& Tomova 1996). The mass of the white dwarf $\left(M_{\mathrm{wd}}\right)$ fitting the EG And models best is $0.4 M_{\odot}$ (Kolb et al. 2004). The mass of the red giant (a M2 III giant) has not been determined. However, we have used $q=3$ which is about the average mass fraction of the red giant to the white dwarf obtained from the literature.

We tested distances ranging from one to ten times the distance between the two stars for the distance between the pumping region and the fluorescent $\mathrm{Fe}^{+}$ions. The distance between the two stars, $a$, based on Eq. (5) which gives $0.7 \mathrm{AU}$, and hence distances spanning from 0.7 to $7 \mathrm{AU}$ between the pumping and fluorescence regions are tested.

$a=\left[\left(\frac{P}{2 \cdot \pi}\right)^{2} \cdot G \cdot(q+1) \cdot M_{\mathrm{wd}}\right]^{\frac{1}{3}}$.

The temperature in the Fe II fluorescence region is assumed to be such that $\mathrm{H}$ I is fully ionized. Further, hydrogen is assumed to be the dominant element of the red giant wind. The electron density in the fluorescence region is therefore estimated based on the expansion velocity of the common envelope $v$, the distance, $D$, and the total mass loss of the system $\dot{M}$ (Eq. (6)). Oliversen et al. (1985) measured the expansion velocity of the common envelope to be $74 \mathrm{~km} \mathrm{~s}^{-1}$. Using this velocity in Eq. (6) limits the electron densities between $1.2 \times 10^{7}$ and $1.2 \times 10^{9} \mathrm{~cm}^{-1}$ for distances $7 \mathrm{AU}$ to $0.7 \mathrm{AU}$, respectively. Since no abundance estimates exist for the nebula we use $\log (\mathrm{Fe}) / \log (\mathrm{H})=-4.5$, which has been used in some of the previous sections. The thickness of the fluorescence region is set to half of the average distance to the pumping region. The calculations are made for all temperatures that give a non-negligible fractions of singly ionized iron.

$n_{\mathrm{e}}=\frac{\dot{M}}{4 \pi v m_{\mathrm{H}} D^{2}}$

With the size of the fluorescence shell, the electron density and the density of iron all locked to the distance between the pumping and fluorescence regions, there are only two free parameters in the calculations of the Fe II fluorescence line intensities, the temperature and the distance between pumping and fluorescence regions. It is possible to obtain observable intensities(based on the $\mathrm{S} / \mathrm{N}$ ratio of the $I U E$ spectra) of the Fe II $\lambda 2493$ line, but not of the $\lambda 2479$ line, for parameters discussed in previous sections. Even though the $\lambda 2479$ line is not observable for any distance or temperature of the fluorescence region, this is not a problem since this line has never been observed in any EG And spectra. According to the calculations, and the fact that the $\lambda 2493$ appears in IUE spectra, the maximum allowed temperature in the Fe II region depends on the distance of the $\mathrm{Fe}^{+}$ions (Fig. 7). The maximum temperature is between 7000 and $9000 \mathrm{~K}$.

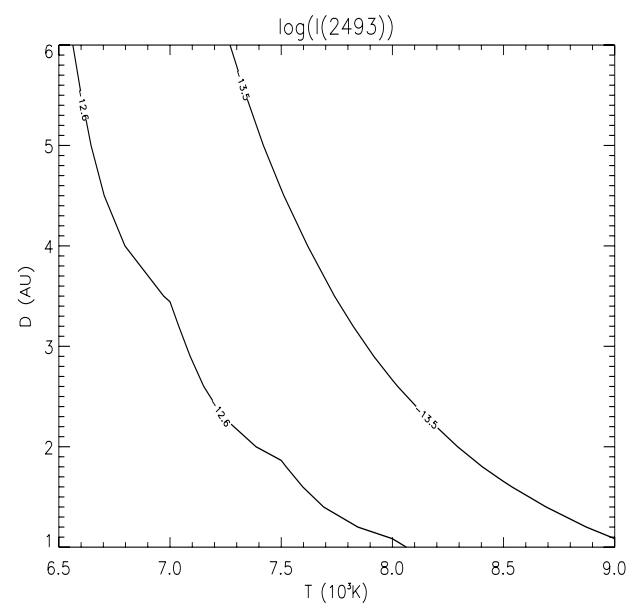

Fig. 7. The contour of the measured $\lambda$ logarithmic intensity $(-12.6)$ in the T-D space. We also plot the -13.5 logarithmic value, which is the lowest possible intensity observable in the lwr04717 spectra. Larger sizes of the common envelope imply lower temperatures.

\subsection{V1016 Cyg}

The orbital period of the binary system V1016 Cyg is not well known. It has been estimated to be around 80 years (Schild \& Schmid 1996), but may be shorter (Parimucha et al. 2002). Most attempts to measure the distance to the system have yielded values from 2 to $10 \mathrm{kpc}$ (Watson et al. 2000). However, Brocksopp et al. (2002) measured the angular separation between the components by imaging, using different filters, to 42 milli arcseconds. For a $2 \mathrm{kpc}$ distance and for approximately solar mass components the minimum orbital period would be 550 years. On the other hand, if the period is 80 years the system would be less than $0.6 \mathrm{kpc}$ from us. Here an orbital period between 80 and 550 years which yields a separation of 20 to $80 \mathrm{AU}$ between the stars will be considered as the possible range.

The region containing the pumped $\mathrm{Fe}^{+}$ions in V1016 Cyg is, as in most D type symbiotic stars, the Mira envelope surrounding the cool component. The radii, temperature and mass used for the fluorescence sphere of V1016 Cyg in this work are set to be the same as for the Mira envelope, as measured by Taranova \& Shenavrin (2000), $6.5 \mathrm{AU}, 2850 \mathrm{~K}$ and 3 to $3.3 M_{\odot}$, respectively. Since Taranova \& Shenavrin (2000) also measured the mass it is possible to obtain an approximate value of the electron density to use for the fluorescence sphere. By assuming $N_{\mathrm{e}}=N_{\mathrm{H}^{+}}$, multiplying both sides of the Saha equation by $n_{\mathrm{e}}$, the electron density can be calculated from Eq. (7) to be around $10^{5} \mathrm{~cm}^{-3}$. The column density obtained from Raman scattering analysis of the cool regions of V1016 Cyg (Jung \& Lee 2004) is $\sim 10^{20} \mathrm{~cm}^{-2}$ which gives an electron density of $10^{6} \mathrm{~cm}^{-3}$, which is ten times higher than that estimated from Eq. (8).

$$
\begin{aligned}
& \left\{\begin{array}{l}
\frac{n_{\mathrm{e}}^{2}}{n_{\mathrm{H}}}=S(T) \\
n_{\mathrm{e}}+n_{\mathrm{H}}=\frac{M}{m_{\mathrm{H}} \cdot V}
\end{array}\right. \\
& n_{\mathrm{e}}=\sqrt{0.25 \cdot S(T)+\frac{M}{m_{\mathrm{H}} \cdot V}}-0.5 \cdot S(T) .
\end{aligned}
$$

In ongoing work, using diagnostics of forbidden lines in the optical region, we have obtained a value of $(5.7 \pm 0.9) \times 10^{5} \mathrm{~cm}^{-3}$ for the electron density in the V1016 Cyg nebulosity. It is clear that the electron density is difficult to obtain and we will consider the range $10^{5}$ to $10^{6} \mathrm{~cm}^{-3}$ in our model. 

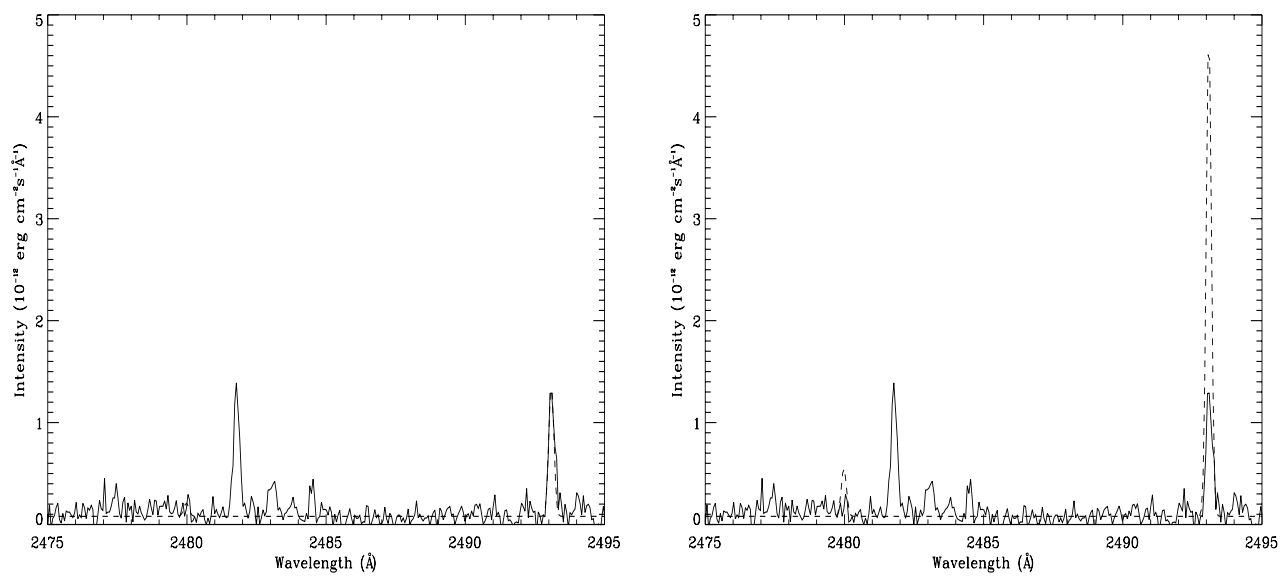

Fig. 8. Fe II fluorescence lines in V1016 Cyg (IUE spectrum lwp04961). In the left, where the model intensity of the $\lambda 2493$ line is set equal to the observed spectrum, the $\lambda 2479$ line is not observable. The projected area covered by Fe II regions relative to the white dwarf in V1016 Cyg would need to be four times as great before any evidence of the Fe II $\mathrm{w}^{2} \mathrm{D}_{3 / 2}$ population would be seen in spectra (right).

Schmid \& Schild (1990) measured the relative iron abundance $(\mathrm{Fe} / \mathrm{H})$ of the V1016 Mira shell to be $10^{-4.6}$. With the mass and volume assumed in the model this yields a number density of iron of $9 \times 10^{4} \mathrm{~cm}^{-3}$. The range of number densities of iron tested for V1016 Cyg is $10^{3}$ to $10^{6} \mathrm{~cm}^{-3}$.

Even though V1016 Cyg is one of the few known symbiotic novae (a small subset of the symbiotic stars that have experienced novae-like outbursts), having erupted in 1964 (Fitzgerald et al. 1966), there is no clear evidence of any white dwarf wind. We therefore do not assume any expansion velocity of the pumping region. The relative velocity structure of the pumping and fluorescent ions is also set to zero in the sampling of V1016 Cyg since the two regions are each associated with one stellar component for which movement relative to each other is mainly perpendicular.

Within the parameter space $\left(n_{\mathrm{e}}, n_{\mathrm{Fe}}, x_{0}\right)$ with ranges mentioned earlier in this section the ratio between the two fluorescing Fe II lines at $\lambda \lambda 2493,2479 \AA I(2493): I(2479)$, ranges from 13 for $n_{\mathrm{Fe}}>10^{5} \mathrm{~cm}^{-3}$ to 20 for $n_{\mathrm{Fe}}=10^{3} \mathrm{~cm}^{-3}$. Since no line is observed at $2479 \AA$ in the spectrum of V1016 Cyg, only the minimum intensity fraction, 6.5 , is calculated from the noise level in the lwp04961 spectrum. Thus, the intensity ratio derived by the model is consistent with the lack of observable Fe II w ${ }^{2} \mathrm{D}_{11 / 2}$ radiation in the spectra of V1016 Cyg. If spectra with a noise level 4 times as good as the lwp04961 spectrum at $2480 \AA$ were available, the calculations predict that radiation from Fe II $\mathrm{w}^{2} \mathrm{D}_{11 / 2}$ would be detectable (Fig. 8).

The calculated integrated intensity of the $\mathrm{Fe} \lambda 2493$ line, $0.00018 \AA^{*}$, which would correspond to $1.4 \times 10^{-14} \mathrm{erg} \mathrm{s}^{-1} \mathrm{~cm}^{-2}$ in the $I U E$ spectra, varies negligibly within the selected parameter space. This is because the fluorescence sphere is optically thick for the channel corresponding to $\left(\mathrm{a}^{4} \mathrm{~F}_{5 / 2}-\mathrm{y}^{4} \mathrm{H}_{11 / 2}\right)$ when the number density of iron exceeds $10^{3} \mathrm{~cm}^{-3}$. The integrated intensity of this line measured in lwp04961 is 26 times as high, $\left(3.7 \times 10^{-13} \mathrm{erg} \mathrm{s}^{-1} \mathrm{~cm}^{-2}\right)$. The only possible way to account for the observed intensity of the Fe II $\lambda 2493$ line is to add clouds of Fe II around the system. However, this means that the Mira shell cannot be the only source of fluorescent $\mathrm{Fe}^{+}$ions in V1016 Cyg.

\section{Summary}

We have attempted to model the strength of two of the strongest PAR-pumped fluorescence lines in seven symbiotic stars. In each case, we constrained our models to lie in a small portion of the total parameter space consistent with the current knowledge of the systems. We were still able to reproduce the correct fluorescence intensities. Additionally, for the symbiotic systems where radiation from the Fe II $\mathrm{w}^{2} \mathrm{D}_{3 / 2}$ level is evident, the fluoroescence was only reproduced by considering a fast outflow containing highly ionized ions. This is consistent with the fact that the Fe II lines from that level have only been observed where white dwarf winds have been or now are confirmed. We suggest that the presence of these lines can be used as indicators of fast outflows in other systems.

Within the unique allowed parameter spaces of the two to three varied parameters (Table 2) there was one small subspace for each symbiotic star for which the observed fluorescence intensities were reproduced. Many characteristics of the structure of symbiotic star nebulae are still unknown and the results from our simple model should be treated with caution. However, we never found more than one subspace to give correct fluorescence intensities for any of our seven cases.

RR Tel: the presence of a white dwarf wind is confirmed and its expansion velocity $\left(500 \mathrm{~km} \mathrm{~s}^{-1}\right)$ is obtained. A fluorescing nebula, enclosing the whole system, with a mean radius of $450 \pm 50 \mathrm{AU}$ is suggested. RW Hya: the presence of a white dwarf wind, already mentioned by Sion et al. (2002), is confirmed and the temperature of the fluorescent part of the nebula of RW Hya is $8200 \pm 400 \mathrm{~K}$. Z And: the $\sim 300 \mathrm{~km} \mathrm{~s}^{-1}$ white dwarf wind suggested by Nussbaumer \& Vogel (1989) must be incorporated into the calculations to explain the observed Fe II fluorescence lines. We conclude that there is (or at least was) a white dwarf wind is this system. Further, the fluorescence occurs in a hot Fe II region ( $T=11-13 \mathrm{kK}$ ) of electron density above $2 \times 10^{9} \mathrm{~cm}^{-3}$. RX Pup: the following ranges in the fluorescing nebula can be given: 3000 to $7000 \mathrm{~K}$ for the temperature and an electron density between $10^{9}$ and $10^{11} \mathrm{~cm}^{-3}$. SY Mus: the nebular temperature is between 4000 and $7500 \mathrm{~K}$. EG And: there are $\mathrm{Fe}^{+}$ions in a spherical or elliptical shell around the binary. However, only approximately half of this shell is fluorescent since the origin of CIV emission, the part of the red giant facing the white dwarf, only "sees" part of the surrounding nebula. V1016 Cyg: an Fe II region in the Mira shell around the cool component is not sufficient to explain the intensity of the fluorescence lines from the $\mathrm{Fe} \mathrm{II}^{4} \mathrm{H}_{11 / 2}$ level. The $\mathrm{Fe}^{+}$ions must be located (or added) in a shell covering most of the system. 


\section{References}

Anandarao, B. G., Taylor, A. R., \& Pottasch, S. R. 1988, A\&A, 203, 361 Baldwin, J. A., Ferland, G. J., Korista, K. T., Hamann, F., \& LaCluyzé, A. 2004, ApJ, 615, 610

Bowen, I. S. 1935, ApJ, 81, 1

Brocksopp, C., Bode, M. F., Eyres, S. P. S., et al. 2002, ApJ, 571, 947

Corradi, R. L. M., Brandi, E., Ferrer, O. E., \& Schwarz, H. E. 1999, A\&A, 343, 841

Dumm, T., Schmutza, W., Schild, H., \& Nussbaumer, H. 1999, A\&A, 349, 169

Dumm, T., Folini, D., Nussbaumer, H., et al. 2000, A\&A, 354, 1014

Eriksson, M., Johansson, S., \& Wahlgren, G. 2001, in Eta Carinae and Other Mysterious Stars: The Hidden Opportunities of Emission Spectroscopy, ed.

T. R. Gull, S. Johannson, \& K. Davidson, ASP Conf. Ser., 242, 325

Eriksson, M., Johansson, S., \& Wahlgren, G. M. 2004, A\&A, 422, 987

Eriksson, M., Johansson, S., Wahlgren, G. M., et al. 2005, A\&A, 434, 397

Eriksson, M., Johansson, S., \& Wahlgren, G. M. 2006, A\&A, 451, 157

Feast, M. W., Glass, I. S., Whitelock, P. A., \& Catchpole, R. M. 1989, MNRAS, 241,375

Ferland, G. J., \& Shields, G. A. 1978, ApJ, 226, 172

Fernandez-Castro, T., Cassatella, A., Gimenez, A., \& Viotti, R. 1988, ApJ, 324, 1016

Fitzgerald, M. P., Houk, N., McCuskey, S. W., \& Hoffleit, D. 1966, ApJ, 144, 1135

Formiggini, L., \& Leibowitz, E. M. 1994, A\&A, 292, 534

Grevesse, N., \& Sauval, A. J. 1999, A\&A, 347, 348

Harper, G. M., Jordan, C., Judge, P. G., et al. 1999, MNRAS, 303, L41

Hartman, H., \& Johansson, S. 2000, A\&A, 359, 627

Hayes, M. A., \& Nussbaumer, H. 1986, A\&A, 161, 287

Imhoff, C. L. 1984, Record of the International Ultraviolet Explorer

NASA/ESA/SERC Three Agency Coordination Meeting, October 3, 4, and 5, 1983, Computer Sciences Corporation, CSC/TM-84/6042, A139

Ivison, R. J., \& Seaquist, E. R. 1994, MNRAS, 268, 561

Johansson, S. 1983, MNRAS, 205, 71P

Jordan, S., Murset, U., \& Werner, K. 1994, A\&A, 283, 475

Jung, Y.-C., \& Lee, H.-W. 2004, MNRAS, 355, 221

Kafatos, M., Michalitsianos, A. G., \& Feibelman, W. A. 1982, ApJ, 257, 204

Kastner, S. O., \& Bhatia, A. K. 1991, ApJ, 381, L59
Kenyon, S. J., \& Fernandez-Castro, T. 1987, ApJ, 316, 427

Kenyon, S. J., \& Mikolajewska, J. 1995, AJ, 110, 391

Kolb, K., Miller, J., Sion, E. M., \& Mikołajewska, J. 2004, AJ, 128, 1790

Kotnik-Karuza, D., Friedjung, M., Whitelock, P. A., et al. 2006, A\&A, 452, 503

Meier, S. R., Kafatos, M., Fahey, R. P., \& Michalitsianos, A. G. 1994, ApJS, 94, 183

Michalitsianos, A. G., Kafatos, M., Fahey, R. P., et al. 1988, ApJ, 331, 477

Mikolajewska, J., \& Kenyon, S. J. 1996, AJ, 112, 1659

Mikolajewska, J., Brandi, E., Hack, W., et al. 1999, MNRAS, 305, 190

Murset, U., Wolff, B., \& Jordan, S. 1997, A\&A, 319, 201

Nussbaumer, H., \& Stencel, R. E. 1987, Exploring the universe with the IUE satellite. (Dordrecht: D. Reidel Publishing Co.), 203

Nussbaumer, H., \& Vogel, M. 1989, A\&A, 213, 137

Oliversen, N. A., Anderson, C. M., Slovak, M. H., \& Stencel, R. E. 1985, ApJ, 295,620

Parimucha, Š., Chochol, D., Pribulla, T., Buson, L. M., \& Vittone, A. A. 2002, A\&A, 391, 999

Penston, M. V., Benvenuti, P., Cassatella, A., et al. 1983, MNRAS, 202, 833

Pereira, C. B., Vogel, M., \& Nussbaumer, H. 1995, A\&A, 293, 783

Proga, D., Mikolajewska, J., \& Kenyon, S. J. 1994, MNRAS, 268, 213

Schild, H., Muerset, U., \& Schmutz, W. 1996, A\&A, 306, 477

Schild, H., \& Schmid, H. M. 1996, A\&A, 310, 211

Schmid, H. M. 2000, in Thermal and Ionization Aspects of Flows from Hot Stars, ed. H. Lamers, \& A. Sapar, ASP Conf. Ser., 204, 303

Schmid, H. M., \& Schild, H. 1990, MNRAS, 246, 84

Schmutz, W., Schild, H., Muerset, U., \& Schmid, H. M. 1994, A\&A, 288, 819

Sikström, C. M., Schultz-Johanning, M., Kock, M., et al. 1999, J. Phys. B Atom. Mol. Phys., 32, 5687

Sion, E. M., Mikołajewska, J., Bambeck, D., \& Dumm, T. 2002, AJ, 123, 983

Skopal, A. 2005, in The Astrophysics of Cataclysmic Variables and Related Objects, ed. J.-M. Hameury, \& J.-P. Lasota, 463

Skopal, A., Vittone, A., \& Errico, L. 1993, Ap\&SS, 209, 79

Stanghellini, L., \& Kaler, J. B. 1989, ApJ, 343, 811

Taranova, O. G., \& Shenavrin, V. I. 2000, Astron. Lett., 26, 600

Tomov, N., \& Tomova, M. 1996, Inf. Bull. Var. Stars, 4341, 1

Vogel, M. 1991, A\&A, 249, 173

Watson, S. K., Eyres, S. P. S., Davis, R. J., et al. 2000, MNRAS, 311, 449

Whitelock, P. A., Menzies, J. W., Evans, T. L., \& Kilkenny, D. 1984, MNRAS, 208, 161 